\title{
CONVEX HULLS OF UNIFORM SAMPLES FROM A CONVEX POLYGON
}

\author{
PIET GROENEBOOM,* Delft University of Technology
}

\begin{abstract}
In Groeneboom (1988) a central limit theorem for the number of vertices $N_{n}$ of the convex hull of a uniform sample from the interior of a convex polygon was derived. To be more precise, it was shown that $\left\{N_{n}-\frac{2}{3} r \log n\right\} /\left\{\frac{10}{27} r \log n\right\}^{1 / 2}$ converges in law to a standard normal distribution, if $r$ is the number of vertices of the convex polygon from which the sample is taken. In the unpublished preprint Nagaev and Khamdamov (1991) a central limit result for the joint distribution of $N_{n}$ and $A_{n}$ is given, where $A_{n}$ is the area of the convex hull, using a coupling of the sample process near the border of the polygon with a Poisson point process as in Groeneboom (1988), and representing the remaining area in the Poisson approximation as a union of a doubly infinite sequence of independent standard exponential random variables. We derive this representation from the representation in Groeneboom (1988) and also prove the central limit result of Nagaev and Khamdamov (1991), using this representation. The relation between the variances of the asymptotic normal distributions of the number of vertices and the area, established in Nagaev and Khamdamov (1991), corresponds to a relation between the actual sample variances of $N_{n}$ and $A_{n}$ in Buchta (2005). We show how these asymptotic results all follow from one simple guiding principle. This corrects at the same time the scaling constants in Cabo and Groeneboom (1994) and Nagaev (1995).
\end{abstract}

Keywords: Convex hull

2010 Mathematics Subject Classification: Primary 60E20

Secondary 49G03; 49F10

\section{Introduction}

Let $N_{n}$ be the number of vertices of the convex hull of a sample of size $n$, drawn uniformly from the interior of a convex polygon with $r$ vertices. It was shown in Groeneboom (1988) that

$$
\left\{N_{n}-\frac{2}{3} r \log n\right\} /\left\{\frac{10}{27} r \log n\right\}^{1 / 2} \stackrel{\mathrm{D}}{\rightarrow} \mathcal{N}(0,1),
$$

where $\mathcal{N}(0,1)$ denotes the standard normal distribution. This was proved by coupling the sample point process near the boundary of the convex polygon with a Poisson point process, and showing that the relevant part of the sample process could be approximated sufficiently closely by the coupled Poisson point process. The central limit result for $N_{n}$ was subsequently derived from a corresponding result for the boundary of the convex hull of the approximating Poisson point process. These methods were also applied to the area $A_{n}$ of the convex hull in Cabo and Groeneboom (1994), but unfortunately the central limit result $A_{n}$ contained a scaling error (see Remark 3.2).

Received 2 November 2010; revision received 11 November 2011.

* Postal address: DIAM, Delft University of Technology, Mekelweg 4, 2628 CD Delft, The Netherlands.

Email address: p.groeneboom@tudelft.nl

Dedicated to the memories of Alexander Nagaev and Tomasz Schreiber. 
Nagaev and Khamdamov (1991), using the coupling of (part of) the sample point process with a Poisson process introduced in Groeneboom (1988), derived the following interesting central limit theorem for the joint distribution of the number of vertices and the area of the convex hull of a uniform sample of $n$ points on the interior of a convex polygon.

Theorem 1.1. (Theorem 1 of Nagaev and Khamdamov (1991).) Let $N_{n}$ denote the number of vertices of the convex hull of a uniform sample of size $n$ from the interior of a convex polygon $C$ with $r \geq 3$ vertices and area $A(C)$. Moreover, let $A_{n}$ denote the area of the convex hull of the sample, and let the scaled 'remaining area' $\bar{A}_{n}$ be defined by

$$
\bar{A}_{n}=\frac{n\left\{A(C)-A_{n}\right\}}{A(C)} .
$$

Then

$$
\left(\frac{10}{27} r \log n\right)^{-1 / 2}\left(N_{n}-\frac{2}{3} r \log n, \bar{A}_{n}-\frac{2}{3} r \log n\right) \stackrel{\mathrm{D}}{\rightarrow} \mathcal{N}(0, \Sigma),
$$

where $\mathcal{N}(0, \Sigma)$ denotes the normal distribution with expectation the zero vector and covariance matrix $\Sigma$ given by

$$
\Sigma=\left(\begin{array}{cc}
1 & 1 \\
1 & \frac{14}{5}
\end{array}\right) .
$$

This is an extension of the central limit theorem for the number of vertices $N_{n}$ in Groeneboom (1988), and one indeed recovers the central limit theorem given there by specializing the above result to the first coordinate. Unfortunately, the preprint Nagaev and Khamdamov (1991), containing this result, was never published. Moreover, it is written in Russian and its length is 50 pages, which might also not have helped its spread in the scientific world.

In a private correspondence Christian Buchta revealed to me that the constant for the central limit theorem for the second component (the remaining area) in Nagaev and Khamdamov (1991) was consistent with a relation he had derived himself between the finite sample variances of $N_{n}$ and $\bar{A}_{n}$.

It is the purpose of the present note to give a simple proof of Theorem 1.1, deriving the result from the central limit theorem for $N_{n}$ in Groeneboom (1988). We think that using the central limit theorem of Groeneboom (1988) considerably simplifies the proof of Theorem 1.1 of Nagaev and Khamdamov (1991) and perhaps more clearly reveals the beauty of their idea. The relation between the variances in Theorem 1.1 can be considered to be a precursor (in an asymptotic sense) of the relation found between the finite sample variances in Buchta (2005).

For recent work on central limit theorems for random polytopes, see, e.g. Bárány and Reitzner (2010a) and Bárány and Reitzner (2010b), where references to earlier work in this area can also be found.

\section{Representation of the remaining area by independent and identically distributed exponentials}

We consider the Poisson point process $\mathcal{P}$ of intensity 1 in $\mathbb{R}_{+}^{2}$, and its left-lower convex hull, as in Groeneboom (1988). To make the connection with Groeneboom (1988), we first restate the definition of the process of vertices $\left\{W(a): a \in \mathbb{R}_{+}\right\}$consisting of the vertices of the (left-lower) convex hull of a Poisson process $\mathcal{P}$ with intensity 1 in $\mathbb{R}_{+}^{2}$.

Definition 2.1. For each $a>0, W(a)=(U(a), V(a))$ is the point of the realization of the Poisson process $\mathcal{P}$ on $\mathbb{R}_{+}^{2}$ such that all points of the realization of $\mathcal{P}$ lie to the right of the line $x+a y=c$ which passes through $W(a)$. If there are several such points (which happens 


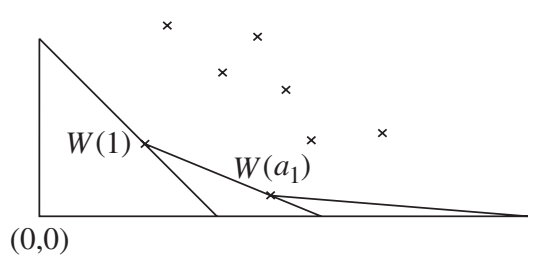

FIGURE 1: The $W(a)$ process.

with probability 0 for fixed $a$ ), we define $U(a)(V(a))$ as the supremum (infimum) of the $x$-coordinates ( $y$-coordinates) of points of this type.

Figure 1 gives a picture of the jump process $W$, where $a_{1}$ is the first jump time after 1 . We now have the following result (see also Theorem 2.1 of Nagaev (1995) for a result of this type).

Theorem 2.1. Let $a_{0}=1$, let $a_{1}, a_{2}, \ldots$ be the jump times of the process $\{W(a): a \geq 1\}$, and let $D_{0}$ be the area of the isosceles triangle $T_{0}$ with a basis, running through $W(1)$, and two equal sides along the $x$ - and $y$-axes, meeting at the top at the origin. Moreover, let $D_{i}, i \geq 1$, be the area of the triangle $T_{i}$, with top at $W\left(a_{i-1}\right)$, basis along the $x$-axis, and sides along the lines $x+a_{i-1} y=U\left(a_{i}\right)+a_{i} V\left(a_{i}\right)$ and $x+a_{i} y=U\left(a_{i}\right)+a_{i} V\left(a_{i}\right)$, where $W\left(a_{i}\right), U\left(a_{i}\right)$, and $V\left(a_{i}\right)$ are defined as in Definition 2.1. Then the following statements hold.

(i) The areas $D_{0}, D_{1}, \ldots$ form an independent and identically distributed (i.i.d.) sequence of standard exponential random variables.

(ii) Let $S_{i}$ be the length of the line segment connecting $W\left(a_{i-1}\right)$ and $W\left(a_{i}\right)$, and let $L_{i}$ be the length of the segment obtained by extending the line segment from $W\left(a_{i-1}\right)$ to $W\left(a_{i}\right)$ until it crosses the $x$-axis. Then the random variables $S_{i}^{2} / L_{i}^{2}, i=1,2, \ldots$, form an i.i.d. sequence of Uniform $(0,1)$ random variables, independent of $W(1)$. Moreover, the $S_{i}^{2} / L_{i}^{2}$ are independent of the sequence $D_{0}, D_{1}, \ldots$

Proof. (i) By Lemma 2.4(i) of Groeneboom (1988) we have, for $z \geq 0$,

$$
\mathrm{P}\left\{D_{0}>z\right\}=\mathrm{P}\left\{\frac{1}{2}\{U(1)+V(1)\}^{2}>z\right\}=\int_{\left\{(x, y):(x+y)^{2} / 2>z\right\}} \mathrm{e}^{-(x+y)^{2} / 2} \mathrm{~d} x \mathrm{~d} y=\mathrm{e}^{-z},
$$

showing that $D_{0}$ has a standard exponential distribution. Let $\mathcal{F}_{a}$ denote the $\sigma$-algebra generated by the points $\{W(b), 1 \leq b \leq a\}$. Then, as shown in Groeneboom (1988), the process of points $\{W(a), a \geq 1\}$ is a Markov process with respect to the filtration $\left\{\mathcal{F}_{a}, a \geq 1\right\}$. Now note that, if $i \geq 1, D_{i}>z$ exactly when there are no points in the triangle of area $z$, with top at $W\left(a_{i}\right)$, basis along the $x$-axis, and sides along the lines $x+a_{i-1} y=U\left(a_{i}\right)+a_{i} V\left(a_{i}\right)$ and $x+a_{i} y=U\left(a_{i}\right)+a_{i} V\left(a_{i}\right)$. Since this event is independent of the location of the points $W\left(a_{0}\right), \ldots, W\left(a_{i-1}\right)$, by the Poisson property of the point process in $\mathbb{R}_{+}^{2}$, we obtain

$$
\mathrm{P}\left\{D_{i}>z\right\}=\mathrm{e}^{-z}, \quad z \geq 0,
$$

where the event $D_{i}>z$ is independent of $D_{0}, \ldots, D_{i-1}$ (note that we can use the strong Markov property here).

(ii) The jump measure $M(a, w ; \cdot)$ of the process $\{W(a): a>0\}$ is given by

$$
M(a, w ; B)=\int_{0}^{y} u \mathbf{1}_{B}(a u,-u) \mathrm{d} u
$$


see Equation (2.22) of Groeneboom (1988). Hence, conditioning on $W(a)=W\left(a_{i-1}\right)=(x, y)$ and the event that there is a jump at time $a$, the location of the next vertex has a density proportional to $u$ (representing the distance of $W(a)$ to the next vertex). So we obtain, for $z \in(0,1)$

$$
\begin{aligned}
\mathrm{P}\left\{\frac{S_{i}^{2}}{L_{i}^{2}}<z \mid W(a)>W(a-)=(x, y)\right\} \\
=\mathrm{P}\left\{S_{i}<L_{i} \sqrt{z} \mid W(a)>W(a-)=(x, y)\right\} \\
=\mathrm{P}\left\{S_{i}<y \sqrt{z\left(1+a^{2}\right)} \mid W(a)>W(a-)=(x, y)\right\} \\
=\frac{2}{y^{2}\left\{1+a^{2}\right\}} \int_{0}^{y \sqrt{z\left(1+a^{2}\right)}} u \mathrm{~d} u \\
=z
\end{aligned}
$$

where we have used the fact that $\frac{1}{2} y^{2}\left\{1+a^{2}\right\}$ is the total measure of the jump measure on the line segment of length $y \sqrt{1+a^{2}}$, connecting $(x, y)$ and $(x+a y, 0)$. This implies that $S_{i}^{2} / L_{i}^{2}$ has a uniform distribution, in accordance with Theorem 2.1 of Nagaev (1995). Moreover, since the distribution neither involves the value of $a=a_{i}$ nor that of $W\left(a_{i-1}\right)$, the sequence of variables $S_{i}^{2} / L_{i}^{2}$ is i.i.d. For the same reason, the variable $S_{i}^{2} / L_{i}^{2}$ is independent of $D_{j}, j \leq i$. It is also seen that $S_{i}^{2} / L_{i}^{2}$ is independent of $D_{j}, j>i$, since the conditional distribution of $D_{i+1}$, given $W\left(a_{i}\right)$, is standard exponential, independently of the value of $W\left(a_{i}\right)$.

Corollary 2.1. Let the sequences $a_{0}, a_{1}, \ldots$ and $V\left(a_{0}\right), V\left(a_{1}\right), \ldots$ be defined as in Theorem 2.1, and let $\tau_{i}=V\left(a_{i}\right) / V\left(a_{i-1}\right), i=1,2, \ldots$ Then the sequence of random variables $\tau_{1}, \tau_{2}, \ldots$ is i.i.d. and

$$
\left(1-\tau_{i}\right)^{2} \sim \text { Uniform }(0,1) .
$$

Moreover, the random variables $\tau_{i}$ are independent of $V\left(a_{0}\right)=V(1)$ and the areas $D_{i}$, where $D_{i}$ is defined as in Theorem 2.1.

Proof. This follows from part (ii) of Theorem 2.1 since

$$
1-\tau_{i}=1-\frac{V\left(a_{i}\right)}{V\left(a_{i-1}\right)}=\frac{V\left(a_{i-1}\right)-V\left(a_{i}\right)}{V\left(a_{i-1}\right)}=\frac{S_{i}}{L_{i}}, \quad i=1, \ldots,
$$

where the last equality is the proportionality relation, well known from elementary geometry.

The following result is the key to Theorem 1.1.

Corollary 2.2. Form $=2,3 \ldots$, let $N(1, m)$ be the number of jumps of the process $\{W(a): a \in$ $[1, m]\}$, and let $[\mathrm{E} N(1, m)]$ be the largest integer smaller than or equal to $\mathrm{E} N(1, m)$. Then the following statements hold.

(i) $\mathrm{E} N(1, m)=\frac{1}{3} \log m$.

(ii) As $m \rightarrow \infty$, the bivariate random variable

$$
\left(\frac{N(1, m)-\mathrm{E} N(1, m)}{\sqrt{5 \log m / 27}}, \sum_{i=1}^{[\mathrm{E} N(1, m)]} \frac{D_{i}-1}{\sqrt{\mathrm{E} N(1, m)}}\right)
$$

converges in distribution to a bivariate normal distribution with expectation 0 and covariance matrix equal to the identity matrix $I$. 
Proof. (i) This is Theorem 2.4(i) of Groeneboom (1988), which is a simple consequence of the fact that the expected jump rate of the process $\{W(a): a \geq 1\}$ is given by $1 /(3 a)$.

(ii) The area $D_{i}$ of the triangle $T_{i}$, as defined in Theorem 2.1 , is given by

$D_{i}=\frac{1}{2} V\left(a_{i-1}\right)\left(V\left(a_{i-1}\right)+a_{i} V\left(a_{i-1}\right)-V\left(a_{i-1}\right)-a_{i-1} V\left(a_{i-1}\right)\right)=\frac{1}{2} V\left(a_{i-1}\right)^{2}\left(a_{i}-a_{i-1}\right)$.

Define

$$
U_{i}=U\left(a_{i}\right), \quad V_{i}=V\left(a_{i}\right), \quad \text { and } \quad W_{i}=\left(U_{i}, V_{i}\right), \quad i=0,1, \ldots
$$

It is clear that (2.1) gives a tridiagonal system for solving $a_{i}$ in terms of the $D_{i}$ and $V_{i}$. We obtain, using $a_{0}=1$,

$$
a_{n}=1+2 \sum_{i=1}^{n} \frac{D_{i}}{V_{i-1}^{2}}, \quad n \geq 1
$$

We now define, for $n \geq 1$,

$$
Y_{n}=V_{n-1}^{2}\left\{1+2 \sum_{i=1}^{n} \frac{D_{i}}{V_{i-1}^{2}}\right\}=V_{n-1}^{2} a_{n}
$$

Thus,

$$
\log a_{n}=-2 \log V_{n-1}+\log Y_{n},
$$

and, hence, we get the 'switching relation':

$$
N(1, m) \geq n \quad \Longleftrightarrow \quad a_{n} \leq m \quad \Longleftrightarrow \quad-2 \log V_{n-1}+\log Y_{n} \leq \log m .
$$

By Corollary 2.1,

$$
\mathrm{E} V_{n}^{2}=\mathrm{E} V_{0}^{2} \prod_{i=1}^{n} \tau_{i}^{2}=6^{-n} \mathrm{E} V_{0}^{2}, \quad \mathrm{E}\left(\frac{V_{n}^{2}}{V_{k}^{2}}\right)=\prod_{i=k+1}^{n} \mathrm{E} \tau_{i}^{2}=6^{-(n-k)}, \quad n>k \geq 0 .
$$

Since, by Theorem 2.1, the $\tau_{i}$ are also independent of the $D_{i}$, we obtain, for all $k \geq 1$,

$$
\begin{aligned}
\mathrm{E} Y_{n} & =6^{-(n-1)} \mathrm{E} V_{0}^{2}+2 \sum_{j=1}^{n} \mathrm{E}\left(\frac{V_{n-1}^{2}}{V_{j-1}^{2}}\right) \\
& =6^{-(n-1)} \mathrm{E} V_{0}^{2}+2 \sum_{j=1}^{n-1} 6^{-j} \\
& \leq 6^{-(n-1)} \mathrm{E} V_{0}^{2}+2 \sum_{j=1}^{\infty} 6^{-j}
\end{aligned}
$$

This implies, by Markov's inequality,

$$
Y_{n}=O_{p}(1) \quad \text { as } n \rightarrow \infty .
$$

Since we also have $Y_{n} \geq 2 D_{n}$ for all $n \geq 1$, where $D_{n}$ has a standard exponential distribution, from this we obtain

$$
\left|\log Y_{n}\right|=O_{p}(1) \quad \text { as } n \rightarrow \infty .
$$


We now obtain, from (2.2),

$$
\frac{\log a_{n}-3 n}{\sqrt{5 n}}=\frac{-2 \log V_{n-1}+\log Y_{n}-3 n}{\sqrt{5 n}}=\frac{-2 \log V_{n-1}-3 n}{\sqrt{5 n}}+O_{p}\left(n^{-1 / 2}\right)
$$

as $n \rightarrow \infty$. Moreover, since

$$
-2 \log V_{n-1}=-2 \sum_{i=1}^{n-1} \log \left(\frac{V_{i}}{V_{i-1}}\right)-2 \log V_{0}=-2 \sum_{i=1}^{n-1} \log \tau_{i}-2 \log V_{0},
$$

we obtain, by the central limit theorem,

$$
\frac{\log a_{n}-3 n}{\sqrt{5 n}}=\frac{-2 \sum_{i=1}^{n-1} \log \tau_{i}-3 n}{\sqrt{5 n}}+o_{p}(1) \stackrel{\mathrm{D}}{\rightarrow} \mathcal{N}(0,1) \quad \text { as } n \rightarrow \infty,
$$

where $\mathcal{N}(0,1)$ denotes the standard normal distribution.

Let

$$
B_{1}(m)=\sum_{i=1}^{[\mathrm{E} N(1, m)]} \frac{\mathscr{D}_{i}-1}{\sqrt{\mathrm{E} N(1, m)}}
$$

and

$$
B_{2}(m)=\frac{N(1, m)-\mathrm{E} N(1, m)}{\sqrt{5 \log m / 27}},
$$

and let, for fixed $y \in \mathbb{R}, n=n_{m, y} \in \mathbb{N}$ be defined by

$$
n=\left[\mathrm{E} N(1, m)+y \sqrt{\frac{5}{27} \log m}\right] \quad \text { as } m \rightarrow \infty .
$$

Then we find, using (2.3) and (2.4), that, as $m \rightarrow \infty$,

$$
\begin{aligned}
\mathrm{P}\left\{B_{1}(m) \geq x, B_{2}(m) \geq y\right\} & \\
& =\mathrm{P}\left\{B_{1}(m) \geq x, N(1, m) \geq \mathrm{E}(1, m)+y \sqrt{\frac{5}{27} \log m}\right\} \\
& \sim \mathrm{P}\left\{B_{1}(m) \geq x, N(1, m) \geq n\right\} \\
& =\mathrm{P}\left\{B_{1}(m) \geq x, \log a_{n} \leq \log m\right\} \\
& =\mathrm{P}\left\{B_{1}(m) \geq x, \frac{\log a_{n}-3 n}{\sqrt{5 n}} \leq \frac{\log m-3 n}{\sqrt{5 n}}\right\} \\
& \sim \mathrm{P}\left\{B_{1}(m) \geq x, \frac{-2 \sum_{i=1}^{n-1} \log \tau_{i}-3 n}{\sqrt{5 n}} \leq \frac{\log m-3 \mathrm{E} N(1, m)-y \sqrt{5 \log m / 3}}{\sqrt{5 n}}\right\} \\
& \sim \mathrm{P}\left\{B_{1}(m) \geq x\right\} \mathrm{P}\left\{\frac{-2 \sum_{i=1}^{n-1} \log \tau_{i}-3 n}{\sqrt{5 n}} \leq-\frac{y \sqrt{5 \log m / 3}}{\sqrt{5 \log m / 3}}\right\} \\
& =\mathrm{P}\left\{B_{1}(m) \geq x\right\} \mathrm{P}\left\{\frac{-2 \sum_{i=1}^{n-1} \log \tau_{i}-3 n}{\sqrt{5 n}} \leq-y\right\},
\end{aligned}
$$

where we have used part (i), (2.5), and Corollary 2.1 (independence of the $\tau_{i}$ and the $D_{i}$ ) in the second to last line. Since, by (2.4),

$$
\mathrm{P}\left\{\frac{-2 \sum_{i=1}^{n-1} \log \tau_{i}-3 n}{\sqrt{5 n}} \leq-y\right\} \rightarrow \Phi(-y)=1-\Phi(y),
$$

where $\Phi$ is the standard normal distribution function, the result now follows. 


\section{The central limit theorem}

In this section we prove a two-dimensional central limit theorem, by combining the results of the preceding section with the results in Groeneboom (1988).

Theorem 3.1. Let $N(a, b)$ be the number of jumps in the interval $[a, b]$ of the process $W$, as defined in Definition 2.1, and let $D(a, b)$ be the area of the union of the triangles $T_{i}$, corresponding to points of jump $a_{i} \in[a, b]$, as defined in Theorem 2.1. Then

$$
\left(\frac{5}{27} \log \left(\frac{b}{a}\right)\right)^{-1 / 2}\left(N(a, b)-\frac{1}{3} \log \left(\frac{b}{a}\right), D(a, b)-\frac{1}{3} \log \left(\frac{b}{a}\right)\right) \stackrel{\mathrm{D}}{\rightarrow} N(0, \Sigma)
$$

as $b / a \rightarrow \infty$, where $N(0, \Sigma)$ is a bivariate normal distribution with expectation 0 and covariance matrix defined by

$$
\Sigma=\left(\begin{array}{cc}
1 & 1 \\
1 & \frac{14}{5}
\end{array}\right)
$$

Proof. As shown by the transformation to a stationary process (see Equation (2.27) of Groeneboom (1988)), the distribution of $N(a, b)$ depends only on the ratio $b / a$. The same construction shows that the distribution of $D(a, b)$ depends only on the ratio $b / a$. So we only have to prove the result for $a=1$ and $b>1$.

We know, from Theorem 2.4 of Groeneboom (1988), that $\mathrm{E} N(1, a)=\frac{1}{3} \log a$ and $\operatorname{var}(N(1, a)) \sim\left(\frac{5}{27}\right) \log a$ as $a \rightarrow \infty$. Moreover,

$$
D(1, a)=\sum_{a_{i} \in[1, a]} D_{i}=\sum_{a_{i} \in[1, a]} \operatorname{area}\left(T_{i}\right),
$$

where the $T_{i}$ are the triangles of Theorem 2.1. So we can consider $D(1, a)$ as a random sum of standard exponential random variables, where the number of terms in the sum is equal to the random variable $N(a, b)$. Reasoning heuristically, as in the case of a compound Poisson distribution, we would obtain

$$
\mathrm{E}(D(1, a))=\mathrm{E} N(1, a)=\frac{1}{3} \log a
$$

and

$$
\operatorname{var}(D(1, a))=\mathrm{E} N(1, a)+\operatorname{var}(N(1, a)) \sim \frac{1}{3} \log a+\frac{5}{27} \log a=\frac{14}{27} \log a .
$$

We now show that we can prove the result by using this heuristic idea.

We write $D(1, a)-\frac{1}{3} \log a$ as the sum of the terms $A_{1}(a)$ and $A_{2}(a)$, where

$$
A_{1}(a)=\sum_{i=1}^{[\mathrm{E} N(1, a)]} D_{i}-\frac{1}{3} \log a
$$

defining $[\mathrm{E} N(1, a)]$ as the largest integer not exceeding $\mathrm{E} N(1, a)=\frac{1}{3} \log a$, and

$$
A_{2}(a)= \begin{cases}\sum_{i=[\mathrm{E} N(1, a)]+1}^{N(1, a)} D_{i} & \text { if } N(1, a)>[\mathrm{E} N(1, a)], \\ -\sum_{i=N(1, a)+1}^{[\mathrm{E} N(1, a)]} D_{i} & \text { if } N(1, a) \leq[\mathrm{E} N(1, a)] .\end{cases}
$$


We now have, if $N(1, a)>[\mathrm{E} N(1, a)]$,

$$
\sum_{i=[\mathrm{E} N(1, a)]+1}^{N(1, a)} D_{i}=\sum_{i=[\mathrm{E} N(1, a)]+1}^{N(1, a)}\left(D_{i}-1\right)+N(1, a)-[\mathrm{E} N(1, a)],
$$

and, similarly, if $N(1, a) \leq[\mathrm{E} N(1, a)]$,

$$
-\sum_{i=N(1, a)+1}^{[\mathrm{E} N(1, a)]} D_{i}=-\sum_{i=N(1, a)+1}^{[\mathrm{E} N(1, a)]}\left(D_{i}-1\right)+N(1, a)-[\mathrm{E} N(1, a)],
$$

where both sides are 0 if $N(1, a)=[\mathrm{E} N(1, a)]$. Hence, we can write

$$
D(1, a)-\frac{1}{3} \log a=A_{1}(a)+N(1, a)-[\mathrm{E} N(1, a)]+R(a),
$$

where

$$
R(a)= \begin{cases}\sum_{i=[\mathrm{E} N(1, a)]+1}^{N(1, a)}\left(D_{i}-1\right) & \text { if } N(1, a)>[\mathrm{E} N(1, a)], \\ -\sum_{i=N(1, a)+1}^{[\mathrm{E}(1, a)]}\left(D_{i}-1\right) & \text { if } N(1, a) \leq[\mathrm{E} N(1, a)] .\end{cases}
$$

Fix $\varepsilon>0$. By Theorem 2.4 of Groeneboom (1988), there exist an $M=M(\varepsilon)>0$ and an $a_{0}=a_{0}(M)$ so that

$$
\mathrm{P}\left\{\left|\frac{N(1, a)-[\mathrm{E} N(1, a)]}{\sqrt{\log a}}\right|>M\right\}<\varepsilon, \quad a \geq a_{0} .
$$

Define

$$
n_{-}(a)=[\mathrm{E} N(1, a)]-M \sqrt{\log a}, \quad n_{+}(a)=[\mathrm{E} N(1, a)]+M \sqrt{\log a} .
$$

Then, by Doob's inequality,

$$
\begin{aligned}
& \mathrm{P}\left\{\max _{m \in\left[[\mathrm{E} N(1, a)]+1, n_{+}(a)\right]}\left|\sum_{i=[\mathrm{E} N(1, a)]}^{m}\left(D_{i}-1\right)\right|>\varepsilon \sqrt{\log a}\right\} \\
& +\mathrm{P}\left\{\max _{m \in\left[n_{-}(a),[\mathrm{E} N(1, a)]\right]}\left|\sum_{i=m}^{[\mathrm{E} N(1, a)]}\left(D_{i}-1\right)\right|>\varepsilon \sqrt{\log a}\right\} \\
& \leq \frac{n_{+}(a)-n_{-}(a)+1}{\varepsilon^{2}(\log a)} \\
& \sim \frac{2 M}{\varepsilon^{2} \sqrt{\log a}} \\
& \quad \rightarrow 0 \quad \text { as } a \rightarrow \infty .
\end{aligned}
$$

These relations imply that $R(a) / \sqrt{\log a}=o_{p}(1)$ and $a \rightarrow \infty$, and, hence,

$$
\frac{D(1, a)-\mathrm{E} N(1, a)}{\sqrt{\log a}}=\frac{\sum_{i=1}^{[\mathrm{E} N(1, a)]}\left(D_{i}-1\right)}{\sqrt{\log a}}+\frac{N(1, a)-[\mathrm{E} N(1, a)]}{\sqrt{\log a}}+o_{p}(1) .
$$

The result now follows from Corollary 2.2 and Theorem 2.4 of Groeneboom (1988). 
Using the methods from Groeneboom (1988) in going from the Poisson approximation to the sample process, we can now easily deduce the central limit result Theorem 1.1 from Theorem 3.1. The latter method is also used in Nagaev and Khamdamov (1991).

Remark 3.1. Instead of working directly with relation (2.1), expressing the differences between successive slopes of the convex hull in terms of the area of the corresponding rectangle and the $y$-coordinate of the vertex at the intersection of the line segments with these slopes, Nagaev and Khamdamov (1991) first wrote this relation in the form

$$
D_{i}=\frac{1}{2} V\left(a_{i-1}\right)^{2}\left(\frac{U\left(a_{i}\right)-U\left(a_{i-1}\right)}{V\left(a_{i-1}\right)-V\left(a_{i}\right)}-\frac{U\left(a_{i-1}\right)-U\left(a_{i-2}\right)}{V\left(a_{i-2}\right)-V\left(a_{i-1}\right)}\right),
$$

and then deduced a recursive relation for the $U\left(a_{i}\right)$ in terms of the $V\left(a_{i}\right)$ and $D_{i}$ from this. They then defined the random time

$$
\theta_{T}=\inf \left\{i: U\left(a_{i}\right) \geq T\right\},
$$

and considered sums of the form $\sum_{i=1}^{\theta_{T}} D_{i}$. This seems to lead to more complicated proofs.

Remark 3.2. The scaling constants for the central limit theorem for the area in Cabo and Groeneboom (1994) are not correct, although a correct application of the methods used in that paper would lead to the central limit theorem for the area, which is part of Theorem 1.1. We here tried to present the results of the unpublished preprint Nagaev and Khamdamov (1991) in an easily understandable way, where the presentation is considerably simplified by the use of martingales, Doob's inequality, and the results from Groeneboom (1988). In view of this simpler approach, and also the fact that Theorem 1.1 is in fact a stronger (two-dimensional) result, this approach seems preferable to the approach in Cabo and Groeneboom (1994). On the other hand, the computations along the lines of Cabo and Groeneboom (1994) give precise information on the first and second moments, as shown below in Section 4.

Although Nagaev (1995) hinted at the proof of Theorem 1.1, there are many important missing steps, which can only be filled in by referring to the unpublished preprint Nagaev and Khamdamov (1991). It seems fair to say that, without knowledge of this preprint, deducing the result from Nagaev (1995) is pretty hard. Moreover, the crucial relation (3.7) of Nagaev (1995) contains an incorrect scaling constant (the constant $\frac{5}{4}$ there should be $\frac{20}{27}$ ), which further complicates the derivation of Theorem 1.1. For this reason, we gave a simplified and selfcontained treatment above.

Remark 3.3. Buchta (2005) gave the following relation between the sample variances of $N_{n}$ and $\bar{A}_{n}$ (using the notation of Theorem 1.1):

$$
\frac{(n+1)(n+2) \operatorname{var}\left(\bar{A}_{n}\right)}{n^{2}}=\operatorname{var}\left(N_{n}\right)+d_{n+2} .
$$

Here

$$
d_{n}=\left(\mathrm{E} N_{n}\right)^{2}-\frac{n\left(\mathrm{E} N_{n-1}\right)^{2}}{n-1}-(2 n-1) \mathrm{E} N_{n}+2 n \mathrm{E} N_{n-1} \sim \mathrm{E} N_{n} \sim \frac{9}{5} \operatorname{var}\left(N_{n}\right)
$$

as $n \rightarrow \infty$. Hence,

$$
\operatorname{var}\left(\bar{A}_{n}\right) \sim \frac{14}{5} \operatorname{var}\left(N_{n}\right) \quad \text { as } n \rightarrow \infty,
$$


in accordance with the covariance matrix $\Sigma$ in Theorem 1 of Nagaev and Khamdamov (1991) (Theorem 1.1 above). Note that the decomposition of the variance of $\bar{A}_{n}$ corresponds to the decomposition (3.1), where $d_{n+2}$ corresponds to the variance of the exponentials $\xi_{i}$ in (3.1) and $\operatorname{var}\left(N_{n}\right)$ corresponds to the variance of the second term on the right-hand side of (3.1).

From Theorem 2 of Buchta (2003), for the number of vertices $N_{n}$ of the convex hull of the points $(0,1),(1,0)$, and $P_{1}, \ldots, P_{n}$, where $P_{1}, \ldots, P_{n}$ is a uniform sample from the interior of the triangle with vertices $(0,0),(0,1)$, and $(1,0)$,

$$
\mathrm{E} N_{n}=\frac{1}{3}\left\{2 \sum_{i=1}^{n} \frac{1}{i}+1\right\}
$$

and

$$
\operatorname{var}\left(N_{n}\right)=\frac{1}{27}\left\{10 \sum_{i=1}^{n} \frac{1}{i}+12 \sum_{i=1}^{n} \frac{1}{i^{2}}-28+\frac{12}{n+1}\right\}
$$

This gives

$$
\mathrm{E} N_{n} \sim \frac{2}{3} \log n, \quad \operatorname{var}\left(N_{n}\right) \sim \frac{10}{27} \log n, \quad \text { as } n \rightarrow \infty,
$$

which corresponds to the distribution results derived in Groeneboom (1988), as is also noted in Buchta (2003).

The results in Groeneboom (1988) and Nagaev and Khamdamov (1991) imply only that a normal limit distribution for the number of vertices of the convex hull of a uniform sample is obtained from the interior of a convex polygon with $r$ vertices by centering with $\frac{2}{3} r \log n$ and dividing by $\left(\frac{10}{27} r \log n\right)^{1 / 2}$. It is not proved there that the variance of the number of vertices itself is also of the order $\frac{10}{27} r \log n$. In principle, it is possible to have a central limit theorem where the scaling needed to obtain the central limit result is different from that obtained from the actual variance. However, the only remaining consideration to go from (3.2) to the result that the variance itself is also of the order $\frac{10}{27} r \log n$ seems to be the appropriate use of the independence of what happens in the corners of the polygons, so that we can conclude that the variance is the sum of the variances of the number of vertices in these corners. Moreover, we have to go from what happens in the triangle to what happens in the corners of the polygon. This is the subject of the current research by Buchta. Results for higher moments of the convex hull of a uniform sample from a triangle with vertices $(0,0),(0,1)$, and $(1,0)$ are given in Buchta (2012).

\section{Simulations}

Let $N(a, b)$ and $D(a, b)$ be defined as in Theorem 3.1. The distribution of these random variables depends only on the ratio $b / a$, and in this section we present some simulation results for these random variables, taking $a=1$ and replacing $b$ by $a$.

The algorithm, given in Section 4 of Nagaev (1995), was used to simulate part of the boundary of the convex hull of a Poisson process with intensity 1 in the first quadrant. The starting triangle is bounded by the $x$-axis, $y$-axis, and a line of the form $x+y=c$, where $c>0$. Its area $D_{0}$ has a standard exponential distribution and the point $W(1)$ is uniformly distributed on the line segment which is the hypotenuse of this triangle.

With the algorithm of Nagaev (1995) we can now generate the points $W(a), a \geq 1$, and simulate in this way the distributions of $N(1, a)$ and $D(1, a)$. We start with $N(1, a)$ and recall 
TABLE 1: Comparison of $\mathrm{E} N(1, a)$ and $\operatorname{var}(N(1, a))$ with simulated and asymptotic values.

\begin{tabular}{rrrrrrr}
\hline \multirow{2}{*}{$\log a$} & \multicolumn{2}{c}{ E $N(1, a)$} & & \multicolumn{3}{c}{$\operatorname{var}(N(1, a))$} \\
\cline { 2 - 3 } \cline { 5 - 6 } & Simulated & Exact & & Simulated & \multicolumn{1}{c}{ Exact } & Asymptotic \\
\hline 10 & 3.3519 & 3.3333 & & 2.1193 & 2.0596 & 1.8519 \\
50 & 16.6668 & 16.6667 & & 9.5908 & 9.4670 & 9.2593 \\
100 & 33.4259 & 33.3333 & & 18.7039 & 18.7263 & 18.5185 \\
\hline
\end{tabular}

TABLE 2: Comparison of $\mathrm{E} D(1, a)$ and $\operatorname{var}(D(1, a))$ with simulated and asymptotic values.

\begin{tabular}{rrrrrrr}
\hline \multirow{2}{*}{$\log a$} & \multicolumn{2}{c}{$\mathrm{E} D(1, a)$} & & \multicolumn{3}{c}{$\operatorname{var}(D(1, a))$} \\
\cline { 2 - 3 } \cline { 5 - 7 } & Simulated & Exact & & Simulated & \multicolumn{1}{c}{ Exact } & Asymptotic \\
\hline 10 & 3.3664 & 3.3333 & & 5.4089 & 5.3040 & 5.1852 \\
50 & 16.6576 & 16.6667 & & 26.1452 & 26.0448 & 25.9259 \\
100 & 33.4933 & 33.3333 & & 52.3304 & 51.9707 & 51.8519 \\
\hline
\end{tabular}

the exact expressions for the expectation $\mathrm{E} N(1, a)$ and $\operatorname{var}(N(1, a))$ from Groeneboom $(1988$, Theorem 2.4):

$$
\begin{gathered}
\mathrm{E} N(1, a)=\frac{1}{3} \log a \\
\operatorname{var}(N(1, a))=\frac{5}{27} \log a+\frac{4}{9}\left(\tan ^{-1}(\sqrt{a-1})\right)^{2}+\frac{8}{9}\left(\frac{\tan ^{-1}(\sqrt{a-1})}{\sqrt{a-1}}-1\right) .
\end{gathered}
$$

As noted at the top of page 34 in Cabo and Groeneboom (1994), the formula for the variance of $N(1, a)$, given in Theorem 2.1 of Groeneboom (1988), contained a typo (the argument of the first $\tan ^{-1}$ above was $a$ instead of $\sqrt{a-1}$ ), and the correct formula is in fact given on page 365 of Groeneboom (1988) (which we use here). Note that these are exact expressions for $\mathrm{E} N(1, a)$ and $\operatorname{var}(N(1, a))$ and not asymptotic ones.

Table 1 shows the means and variances for 10000 simulations for $\log a=10,50$, and 100 . The exact values are given to four decimal places.

It is seen from Table 1 that $\mathrm{E} N(1, a)$ and $\operatorname{var}(N(1, a))$ are quite close to the simulated values and that, not unexpectedly, for $a=10$, the exact expression for the variance of $N(1, a)$, given by (4.1), is closer to the simulated value than the asymptotic value.

Similarly, we performed 10000 simulations for $\log a=10,50$, and 100 to simulate the behavior of $D(1, a)$. Using the (corrected) methods of computation of Cabo and Groeneboom (1994) (details are given in Groeneboom (2011b)), it can be shown that

$$
\mathrm{E} D(1, a)=\frac{1}{3} \log a
$$

and, defining $\alpha=a-1$, that

$$
\begin{aligned}
\operatorname{var}(D(1, a))= & \frac{14}{27} \log a+\frac{2}{3 \alpha^{2}}+\frac{4}{9 \alpha}-\frac{44}{45}-\frac{2\{3+\alpha(3-4 \alpha)\} \tan ^{-1}(\sqrt{\alpha})}{9 \alpha^{5 / 2}} \\
& +\frac{4}{9}\left(\tan ^{-1}(\sqrt{\alpha})\right)^{2} .
\end{aligned}
$$

These are again exact expressions for $\mathrm{E} D(1, a)$ and $\operatorname{var}(D(1, a))$ and not asymptotic ones. The results are given in Table 2. 
TABLE 3: Comparison of $\mathrm{E} v_{t}$ and $\operatorname{var}\left(v_{t}\right)$ with simulated and asymptotic values.

\begin{tabular}{rrrrrrr}
\hline \multirow{2}{*}{$\log t$} & \multicolumn{2}{c}{$\mathrm{E} v_{t}$} & & \multicolumn{3}{c}{$\operatorname{var}\left(v_{t}\right)$} \\
\cline { 2 - 3 } \cline { 5 - 7 } & Simulated & Exact & & Simulated & $\frac{20}{27} \log t$ & $\frac{5}{4} \log t($ Nagaev $(1995))$ \\
\hline 10 & 13.0778 & 13.3333 & & 7.2630 & 7.40741 & 12.5 \\
50 & 66.4792 & 66.6667 & & 37.6192 & 37.0370 & 62.5 \\
100 & 133.1330 & 133.3333 & & 74.542 & 74.0741 & 125 \\
\hline
\end{tabular}

We finally turn our attention to Relation (3.7) of Nagaev (1995). This relation gives asymptotic expressions for the expectation and variance of the number, $v_{t}$, of vertices falling in a disk $S_{t}$ with radius $t$ and center $(0,0)$. On the basis of the results in Groeneboom (1988), it is to be expected that

$$
\mathrm{E} v_{t} \sim \frac{4}{3} \log t, \quad \operatorname{var}\left(v_{t}\right) \sim \frac{20}{27} \log t, \quad \text { as } t \rightarrow \infty,
$$

whereas Relation (3.7) of Nagaev (1995) gives the above relation for $\mathrm{E} v_{t}$, but $\frac{5}{4} \log t$ as the asymptotic expression for $\operatorname{var}\left(v_{t}\right)$. The argument for (4.2) is that, first of all, $v_{t}$ can be expected to behave asymptotically as the number of vertices with coordinates $x>y$ such that $x<t$ plus the number of vertices with coordinates $y \geq x$ such that $y<t$, since vertices with large $x$-coordinates will, with high probability, be very close to the $x$-axis and vertices with large $y$-coordinates will, with high probability, be very close to the $y$-axis. Secondly, again by Groeneboom (1988), the number of vertices with coordinates $x>y$ such that $x<t$ will behave asymptotically as $N\left(1, t^{2}\right)$, and, similarly, the number of vertices with coordinates $y \geq x$ such that $y<t$ will behave asymptotically as $N\left(1 / t^{2}, 1\right)$.

By the construction of the algorithm in Nagaev (1995), we can simulate the number of vertices $W(a), a \geq 1$, satisfying $U(a)^{2}+V(a)^{2}<t^{2}$, by running the algorithm till we obtain a vertex $W(a)$ such that

$$
U(a)^{2}+V(a)^{2} \geq t^{2}
$$

The resulting asymptotic behaviors of $\mathrm{E} v_{t}$ and $\operatorname{var}\left(v_{t}\right)$ are obtained from this by multiplying the results by the factor 2 . In Table 3 we present the results for 10000 simulations for $\log t=10$, 50 , and 100 .

Table 3 clearly suggests that the factor $\frac{5}{4}$ is much too large and that the correct approximation is indeed given by (4.2).

\section{Concluding remarks}

There is a remarkable analogy between the behavior of the left-lower convex hull of the Poisson point process, discussed above, and the least concave majorant of (one-sided) Brownian motion without drift, as analyzed in Groeneboom (1983). In the same way there is an analogy between the behavior of the lower convex hull of the Poisson point process inside a parabola, as analyzed in Groeneboom (1988) and Nagaev (1995), and the least concave majorant of Brownian motion with a parabolic drift, as studied in Groeneboom (1989) and Groeneboom (2011a). Why this is the case is still somewhat of a mystery and deserves (in my view) further investigation. 


\section{Acknowledgements}

I would like to posthumously thank Tomasz Schreiber for sending me the unpublished preprint Nagaev and Khamdamov (1991), and Christian Buchta for making me aware of Buchta (2003) and sending me the preprint Buchta (2012).

\section{References}

Bárány, I. and Reitzner, M. (2010a). Poisson polytopes. Ann. Prob. 38, 1507-1531.

Bárány, I. AND ReITZner, M. (2010b). On the variance of random polytopes. Adv. Math. 225, 1986-2001.

Buchta, C. (2003). On the distribution of the number of vertices of a random polygon. Anz. Österreich. Akad. Wiss. Math. Natur. Kl. 139, 17-19.

Buchta, C. (2005). An identity relating moments of functionals of convex hulls. Discrete Comput. Geom. 33, $125-142$.

Buchta, C. (2012). On the boundary structure of the convex hull of random points. Adv. Geom. 12, 179-190.

Cabo, A. J. and Groeneboom, P. (1994). Limit theorems for functionals of convex hulls. Prob. Theory Relat. Fields 100, 31-55.

Groeneboom, P. (1983). The concave majorant of Brownian motion. Ann. Prob. 11, 1016-1027.

Groeneboom, P. (1988). Limit theorems for convex hulls. Prob. Theory Relat. Fields 79, 327-368.

Groeneboom, P. (1989). Brownian motion with a parabolic drift and Airy functions. Prob. Theory Relat. Fields 81, 79-109.

Groeneboom, P. (2011a). Vertices of the least concave majorant of Brownian motion with parabolic drift. Electron. J. Prob. 16, 2234-2258.

Groeneboom, P. (2011b). The remaining area of the convex hull of a Poisson process. Preprint. Available at http:// arxiv.org/abs/1111.2504v2.

Nagaev, A. V. and Khamdamov, I. M. (1991). Limit theorems for functionals of random convex hulls. Preprint. Institute of Mathematics, Academy of Sciences of Uzbekistan.

Nagaev, A. V. (1995). Some properties of convex hulls generated by homogeneous Poisson point processes in an unbounded convex domain. Ann. Inst. Statist. Math. 47, 21-29. 